\title{
Who Makes the Diagnosis? A Retrospective Observational Study Comparing the Emergency Department Initial Diagnosis and the Internal Medicine Discharge Diagnosis
}

Filomena Pietrantonio ${ }^{1^{*}}$ and E. Scotti ${ }^{2}$

${ }^{1}$ Internal Medicine Unit, Manerbio Hospital Manerbio (BS), ASST-Garda, Italy

${ }^{2}$ Acute Internal Medicine Unit, INI Research Insitute and Clinic, Grottaferrata (Rome), Italy

\begin{abstract}
Background: Despite the central role of Internal Medicine (IM) in emergency admission management, both users and health planners do not seem to recognize the distinct features of the activities relative to IM. According to the Literature, the role of IM is characterized by: (1) Acute, critical, multiple pathology and complex patient management; (2) Difficult clinical diagnosis; (3) Individuation of priorities; (4). Hospitalterritory pathways promoting integration of diverse specialist activities.
\end{abstract}

Objective: To determine the proportion of correct and missed emergency department (ED) diagnoses compared to IM discharge diagnoses.

Methods: ED diagnoses and hospital IM discharge diagnoses were compared. By using the consensus among experts method a diagnosis evaluation grid was formed. Diagnosis was defined as follows: (1) The "gold standard" diagnosis (correct diagnosis), according to ICD10 (10th International Classification of Diseases and Related Health Problems), independently made by two experienced IM specialists and reported in the discharge letter. (2) ED diagnosis made by the Emergency Physician and reported in the patient acceptance or transfer record to the Internal Medicine Unit; (3) Priority Error: the correct diagnosis appears as a secondary diagnosis in the ED diagnosis; (4) Incomplete diagnosis: diagnostic orientation without a precise diagnosis; (5) Diagnosis Error: the correct diagnosis was not made in the ED. The first 13 diagnoses made in ED are defined as the most frequent in number within the sample being examined.

Results: 317 non trauma patients presenting to the ED from June to September 2016 and admitted to the INI (Italian Neurotraumatology Institute) IM department were included for final analysis. The final diagnosis at IM discharge was taken to be the correct "gold standard" diagnosis. In 180 patients $(56,7 \%)$ this corresponded with the primary ED diagnosis, in 104 patients $(32,8 \%)$ the diagnosis was missed and in the remaining $10.5 \%$, the diagnosis (33 patients) was incomplete or a priority error occurred. The most frequent final diagnoses were cardiac failure $(n=53)$, pneumonia $(n=43)$, TIA (Transient Ischemic Attack) $(n=31)$; respiratory failure $(n=28)$; COPD (Chronic Obstructive Pulmonary Disease) $(n=21)$, correctly diagnosed in the ED in 37, 26, 19, 20, and 11 patients, respectively.

Conclusion: Patients presenting to ED with acute symptoms represent a diagnostic challenge that in $43.3 \%$ of cases is explained by the clinical activity carried out by the Internal Medicine specialist during hospitalization. The study confirms the central role of Internal Medicine in defining the correct diagnosis in acute and complex patients. It is likely time to instigate awareness campaigns for patients and policy makers promoting the central role of Internal Medicine in hospital organization and hospital-territory integration and to duly recognize the complexity of IM activity through the endorsement of appropriate DRGs (Diagnosis Related Groups) in the Medical Area.

\section{Background}

The Emergency Department (ED) serves as a hub for prehospital emergency medical systems, as an acute diagnostic and treatment center, a primary safety net, and a $24 / 7$ portal for rapid inpatient admission[1]. Worldwide the ED mainly plays a filter role by hospitalizing only those who actually require admission while referring the more than $80 \%$ of presenters to other care settings. The entire Emergency organization was based on severity codes assigned by triage, however in reality clinical status tends to evolve over time, meaning output codes may be very different from entry codes. The hospital organization downstream of the ED was then built on the entry codes, not reflecting the reality of output codes, particularly for critical patients, meaning triage assigned codes did not correspond with admitted patients' codes. Moreover, ED is ideated to stabilize critical patients, more so than finding the correct etiological diagnosis. The third function played by ED is to differentiate medical from surgical patients, directing them towards the appropriate specialty in consideration of the gravity of their condition. Complex patients with a single pathology are sent to an organ/system specific unit but they represent a minority, as most patients present acutely with a complex array of clinical issues, and are thus destined for the Internal Medicine Unit. The emergency physician is not required to conduct a complete etiological and exhaustive diagnosis but draws

${ }^{*}$ Corresponding Author: Dr. Filomena Pietrantonio, Internal Medicine Unit, Manerbio Hospital Manerbio (BS), Brescia, ASST-Garda, Italy; Email: filomena.pietrantonio@gmail.com

Citation: Pietrantonio F, Scotti E (2018) Who Makes the Diagnosis? A Retrospective Observational Study Comparing the Emergency Department Initial Diagnosis and the Internal Medicine Discharge Diagnosis. Int J Community Fam Med 3: 137. https://doi.org/10.15344/2456-3498/2018/137

Copyright: (c) 2018 Pietrantonio, et al. This is an open-access article distributed under the terms of the Creative Commons Attribution License, which permits unrestricted use, distribution, and reproduction in any medium, provided the original author and source are credited. 
Citation: Pietrantonio F, Scotti E (2018) Who Makes the Diagnosis? A Retrospective Observational Study Comparing the Emergency Department Initial Diagnosis and the Internal Medicine Discharge Diagnosis. Int J Community Fam Med 3: 137. https://doi.org/10.15344/2456-3498/2018/137

Page 2 of 5

provides a working differential which aids in directing further diagnostic and therapeutic choices in the inpatient setting, where the can work started in ED can be concluded. The Department of Emergency Medicine also responds to this logic of stabilization, initialization and subsequent transfer to the proper care setting.

In addition, it is important to consider the proportion of elderly patients with multiple pathologies, with complex social problems who are unable to be treated at home and go to the ED not only health problems but also social assistance.

Despite the central role of Internal Medicine (IM) in emergency admissions management, both users and health planners do not seem to recognize the distinct features of IM services. According to the literature [2], the role of IM is characterized by: (1) Acute, critical, multiple pathology and complex patient management [3]; (2) Difficult clinical diagnosis; (3) identification of priorities; (4) Hospital-territory pathways promoting integration of diverse specialist activities. [4].

The new model based on intensity of care currently used in IM Departments has the ambitious goal of achieving the best clinical efficacy and assistance while ensuring a sustainable economic commitment [5]. Patient stratification begins in the Emergency Department with correct assessment and diagnosis, supported by a stepped care organizational scheme guiding patient management following an integrated medical and nursing assessment based on clinical condition and the complexity of care. The applicability of a model according to intensity of care: (i) requires flexibility in consideration of the structure in which it occurs; (ii) generates different organizational structures; (iii) each level requires adequate presence of medical/nursing staff and technology; (iv) different pathways have to be provided depending on the characteristics of the patients; (v) in the definition of responsibilities a central role is given to the hospital internist [6].

Diagnosis is a crucial point in this model, however review of the literature revealed very little evidence in terms of the effective figure making the diagnosis in patients presenting to the ED with acute symptoms.

The objective of the current study is to determine the proportion of correct and missed Emergency Department diagnoses compared to Internal Medicine discharge diagnoses through a retrospective observational study conducted at the INI (Neurotraumatology Institute-Grottaferrata-RM) Internal Medicine Department.

\section{Methods}

\section{Study design}

This retrospective observational study was conducted from June to September 2016. Since this is an observational retrospective study the Institutional Review Board (IRB)/ Independent Ethics Committees (IEC) acknowledged and approved the study. The study was performed in the Internal Medicine Department at INI (Neurotraumatology Institute-Grottaferrata-RM), a 225-bed private facility accredited by the National Health Service, which receives patients from the main South-West Rome Hospitals' Emergency Departments (Tor Vergata University, Albano, Velletri, Tivoli, Frascati, Palestrina Hospitals). All adult (i.e. $\geq 18$ years) non trauma patients who presented to the ED with complaints related to medical diseases were eligible. Patients referred from other hospitals, patients who brought results of recent blood tests to the ED, falsely enrolled patients with specific complaints or patients with vital parameters markedly out of range (systolic blood pressure $<80 \mathrm{~mm} \mathrm{Hg}$, heart rate $>120$ beats/minute, temperature $>38.4^{\circ} \mathrm{C}$ or $<35.6^{\circ} \mathrm{C}$, respiratory rate $>30 \mathrm{breaths} /$ minute), patients who needed surgery, patients who did not sign the informed consent form, and patients within complete data were excluded. All patients were enrolled consecutively, 24 hours a day, by a study team.

\section{Measurements and Outcomes}

Three previously trained study physicians recorded the following data on the patients' case report forms shortly after admission: demographic baseline data, vital signs (pulse, blood pressure, respiratory rate, and oxygen saturation), all complaints (using a structured interview form), Glasgow Coma Scale score, medical history, physical examination, and electrocardiography. Venous blood samples were taken from all patients; chest $\mathrm{X}$-rays and urinalysis were performed at the discretion of the treating physician. The outcome measures were (i) the proportion of correct diagnoses made at the ED; (ii) the proportion of correct hospital discharge diagnoses compared with the proportion of missed diagnoses.

\section{Definitions}

\section{Gold standard diagnosis}

Final diagnosis, according to rules of the $10^{\text {th }}$ International Classification of Diseases and Related Health Problems (ICD10), made by two Internal Medicine Specialists at IM discharge. Information required to establish the gold standard diagnosis was collected from the patients' general practitioners or from hospital discharge reports. The gold standard diagnosis was established by two independent physicians with longstanding experience, certified in Internal Medicine. In the case of disagreement, patient records were reviewed and consensus was reached by an expert panel, consisting of two physicians certified in Internal Medicine and one physician certified in Emergency Medicine, with at least 10 years of experience.

\section{ED diagnoses (primary, i.e. first listed)}

The ED diagnosesthat were made by the emergency physicians at transfer from the ED.

\section{Correct Diagnosis}

Primary ED diagnosis was identical to the gold standard diagnosis.

\section{Hierarchy problem}

The gold standard diagnosis was listed as a secondary ED diagnosis, but not as the primary ED diagnosis.

\section{Missed diagnosis}

The gold standard diagnosis was not made in the ED. If the primary ED diagnosis was a narrow differential diagnosis compatible with the gold standard diagnosis it was classified as a correct diagnosis. If, however, the gold standard diagnosis was one of a large range of varying ED differential diagnoses, it was classified as a missed diagnosis. 
Citation: Pietrantonio F, Scotti E (2018) Who Makes the Diagnosis? A Retrospective Observational Study Comparing the Emergency Department Initial Diagnosis and the Internal Medicine Discharge Diagnosis. Int J Community Fam Med 3: 137. https://doi.org/10.15344/2456-3498/2018/137

Page 3 of 5

\section{Results}

317 non trauma patients presenting to the ED from June to September 2016 and admitted to the INI (Italian Neurotraumatology Institute) IM department were included for final analysis. The final diagnosis at IM discharge was taken to be the correct "gold standard" diagnosis. In 180 patients $(56,7 \%)$ this corresponded with the primary ED diagnosis, in 104 patients $(32,8 \%)$ diagnosis was missed and in the remaining $10.5 \%$ (33 patients) the diagnosis was incomplete or a priority error occurred. The most frequent final diagnoses were cardiac failure $(n=53)$, pneumonia $(n=43)$, TIA (Transient Ischemic Attack) $(n=31)$; respiratory failure $(n=28)$; COPD (Chronic Obstructive Pulmonary Disease) $(n=21)$, correctly diagnosed in the ED in $37,26,19,20$, and 11 patients, respectively.

The study results are showed in Table 1 .

The primary ED diagnosis was correct in $180(56,7 \%)$ patients, and a hierarchy problem occurred in 6 patients. Table 1 shows the 13 most common diagnoses in the ED, representing a total of 281 patients. In this group of patients, a total of 170 (60\%) diagnoses were correct. The most often missed conditions were Chronic Vascular Encephalopathy, Syncope, Hyponatremia. The three most common diagnoses identified were Cardiac Failure (18\%), Pneumonia (15\%), and Respiratory Failure (10\%)

\section{Limitation of the study}

Our study had several limitations. Firstly, the characteristics of ED physicians and physicians working on the wards were not assessed. Thus, the influence of factors such as the physician's age and experience was not included in the analysis. Secondly, we assessed correct diagnosis as an outcome, but not mortality. Thus, we are not able to determine the influence on mortality of making a wrong diagnosis or of missing diagnoses. However, current quality standards in our healthcare system require a diagnosis for every patient seen in the ED. Moreover, DRGs (Diagnosis Related Groups) require a diagnosis to discharge hospitalized patients. Thus, correct diagnosis might be an interesting outcome, even though mortality was not assessed. Finally, this was a single centre study and our study population mainly consisted of elderly patients. Therefore, our results have to be confirmed by further studies before generalizing.

\section{Discussion}

Comparing ED reports with Internal Medicine Unit discharge letters, and considering the gold standard diagnosis as that reached at the conclusion of a clinical itinerary supported by specific tests and application of evidence-based guidelines highlighted the diagnostic role of Internal Medicine Unit. In fact, in $56,7 \%$ of patients the final diagnosis matched the ED diagnosis, while in $32,8 \%$ of patients the ED diagnosis was proved wrong. The most frequent final diagnoses were: Heart Failure, Pneumonia, TIA, respiratory failure, COPD, Stroke, Cirrhosis, in line with the most frequent DRGs in Internal Medicine at the national level [7].

The study results agree with evidence reported in the literature. Arno et al. [8], in a prospective observational study comparing diagnoses made in the ED, demonstrated that hospital discharge diagnoses and final diagnoses corresponded with the primary ED diagnosis in $263(46.0 \%)$ and to the hospital discharge diagnosis in 292 (51\%) of patients. The most frequent final diagnoses were urinary tract infections, electrolyte disorders, and pneumonia.

In this study we found that patients presenting to the ED with acute symptoms represent a diagnostic challenge that in $43.3 \%$ of cases is only explained as a result of the clinical activity of the Internal Medicine specialist during hospitalization. Moreover, the study confirms the

\begin{tabular}{|c|c|c|c|c|c|c|c|}
\hline Diagnosis & $\begin{array}{c}\text { Discharge diagnosis } \\
\text { (from IM Department) } \\
\text { Gold standard (correct) }\end{array}$ & $\begin{array}{l}\text { Emergency Department } \\
\text { correct diagnosis (equal } \\
\text { to Gold standard) }\end{array}$ & $\begin{array}{c}\text { Correct } \\
(\%)\end{array}$ & $\begin{array}{c}\text { Wrong } \\
(\%)\end{array}$ & $\begin{array}{l}\text { Priority } \\
\text { Error }\end{array}$ & $\begin{array}{l}\text { Incomplete } \\
\text { diagnosis }\end{array}$ & Wrong \\
\hline 1. Cardiac failure & 53 & 37 & 69 & 20 & & 5 & 11 \\
\hline 2. Pneumonia & 43 & 26 & 60 & 30 & 1 & 3 & 13 \\
\hline 3. TIA & 31 & 19 & 61 & 25 & & 4 & 8 \\
\hline 4. Respiratory Failure & 28 & 20 & 71 & 0,03 & 2 & 5 & 1 \\
\hline 5. COPD & 21 & 11 & 52 & 23 & 2 & 3 & 5 \\
\hline 6. Fever & 18 & 5 & 27 & 61 & 1 & 1 & 11 \\
\hline 7. Stroke & 15 & 12 & 80 & 0,06 & & 2 & 1 \\
\hline 8. Cirrhosis & 15 & 12 & 80 & 20 & & & 3 \\
\hline 9. Syncope & 15 & 7 & 46 & 54 & & & 8 \\
\hline 10. Urinary tract infection & 14 & 8 & 57 & 36 & & 1 & 5 \\
\hline $\begin{array}{l}\text { 11. Dementia/chronicva } \\
\text { scularenc ephalopaty }\end{array}$ & 13 & 4 & 30 & 70 & & & 9 \\
\hline 12. Dehydration & 8 & 5 & 62 & 38 & & & 3 \\
\hline 13. Hyponatremia & 7 & 4 & 57 & 43 & & & 3 \\
\hline Total & 281 & 170 & $60 \%$ & $29 \%$ & 6 & 24 & 81 \\
\hline Other diagnosis & 36 & 10 & & & & 3 & 23 \\
\hline GENERAL TOTAL & 317 & 180 & $56,7 \%$ & $32,8 \%$ & & 27 & 104 \\
\hline
\end{tabular}

Table 1: the most frequent Emergency department diagnosis. 
Citation: Pietrantonio F, Scotti E (2018) Who Makes the Diagnosis? A Retrospective Observational Study Comparing the Emergency Department Initial Diagnosis and the Internal Medicine Discharge Diagnosis. Int J Community Fam Med 3: 137. https://doi.org/10.15344/2456-3498/2018/137

Page 4 of 5

central role of Internal Medicine in defining the correct diagnosis in acute and complex patients. It is likely time therefore, to replace the title of Internal Medicine with another designation, such as, for example, Diagnostic Medicine, which defines more appropriately the current role played by this specialist branch. This has previously been described by Wahner-Roedler et al. [9] who, in a retrospective study, demonstrated that 246 out of 248 patients received a final diagnosis during General Internal medicine hospitalization. The diagnoses were made by relying on the clinical judgment of the General Internist in $50.4 \%$ of cases, following a radiology study in $31.7 \%$, a blood test or culture result in $9.4 \%$, biopsy findings in $3.3 \%$ and various other diagnostic studies (endoscopy, echocardiography, electromyography and electroencephalography) in $5.2 \%$. Clinicians provided the correct diagnosis significantly more often than radiologic studies $(\mathrm{P}=0.0015)$, which was the next most useful type of diagnostic evaluation.

However, few studies in the literature deal with diagnostic errors, though an important field in the improvement of quality of care. Interesting outcomes are highlighted in the work of Shiff et al. A total of 669 cases were reported by 310 clinicians from 22 institutions participating in a written survey. Errors occurred most frequently in the testing phase (failure to order, report, and follow-up laboratory results) (44\%), followed by clinician assessment errors (failure to consider and weigh up competing diagnosis) (32\%), history taking (10\%), physical examination (10\%), and referral or consultation errors and delays (3\%).

The low rate of correct diagnoses in the ED may be the result of various factors. As reported previously, the ED filter role, the broad spectrum of underlying diagnoses and difficulties in history taking in an overcrowded ED present challenges for Emergency Physicians, and may therefore explain the low rate of correct diagnoses $[11,12]$. On the other hand, it is conceivable that the ward physicians could be influenced by wrong diagnoses given by the Emergency Physicians, and continue to miss primary diagnoses [13]

\section{Defining Internal Medicine patient}

The question: "who is the Internal Medicine patient?" needs a simple and comprehensible answer. The main role of the Internal Medicine specialist is to delineate complex diagnoses, stabilization, prioritization, prognostic stratification and hospital-territory integration [14]. Moreover, numerous social assistance-related activities are performed in Medical Units, sometimes compensating those lacking in other care settings outside of the hospital. These activities mainly concern fragile, multiple-pathology patients with loss of functional autonomy and other social, economic, health, logistical and familial problems, necessitating continued health care in acute hospital wards, in the absence of solutions in outpatients care settings.

The task of the hospital Internal Medicine specialist does not end when severely and acutely ill patients, with complex and multiple pathologies, are stabilized, but rather lies in the difficult and detailed etiological diagnoses, working his or her way through different symptoms, signs and clinical care problems of all kinds. To achieve this, the internist must have ample experience and extensive training covering all fields of medicine. In other words, the internist must take a holistic view and consider the human body as a whole unit that cannot be expressed by a summing up of the parts. With this approach, the measurement of body function is always greater than (and different to) the sum of the performance of the individual parts. The human body can, therefore, represent an integrated system according to which the function of each organ influences that of the other organs, and disease or dysfunction of one has significant repercussions on the others. As a consequence of epidemiological transition, in Italy, as in other Developed Countries, a large share of economic resources is absorbed by the management of chronic conditions. Up to $65-70 \%$ of health care expenditure goes to treating chronic diseases in different phases of illness [15]. The global view provided by the internist permits identification of priorities and choice of the most direct path to making a diagnosis while saving in time and money.

The activity of the Internist reflects the capacity to allocate patients to an appropriate care setting at different stages of the illness as a function of the disease process. The pathway comprises: (i) a phase of acute patient stabilization and stratification which occurs in the Emergency Department and concludes with the initial establishment of an in hospital patient path; (ii) the hospital response process involving the Internal Medicine unit, regarding overall management of the internal medicine patients, identifying priorities, monitoring progress and determining the final diagnosis; iii) integration with the specialists in other fields to define the next steps in the patients' clinical path and to choose different care settings after the acute phase according to the patients' needs[16].

Moreover, the diagnostic role of the IM Department is highlighted by the significant percentage of missed primary ED diagnoses corrected at discharge [17]

\section{Conclusion}

The main outcomes of this study are represented by the evidence that diagnosis is one of the main activities of the Internal Medicine specialist and the Emergency department's role is to filter, to stabilize and to stratify patients. Patients presenting in the ED with acute symptoms represent a diagnostic challenge resolved in $43,3 \%$ of the cases by the IM specialist. Finally, the epidemiological transition has determined a rising number of chronically ill patients that are putting the health care system under increasing stress, and as such require the specialist of clinical complexity, the internist, in order to guarantee appropriate treatment. The study confirms the central role of Internal Medicine in determining the correct diagnosis in acute and complex patients. It is likely time to instigate awareness campaigns for patients and policy makers promoting the central role of Internal Medicine in hospital organization and hospital-territory integration and to duly recognize the complexity of IM activity through the endorsement of appropriate DRGs (Diagnosis Related Groups) in the Medical Area.

\section{Conflict of Interest}

No authors have a conflict of interest or any financial tie to disclose.

\section{References}

1. Schuur JD, Venkatesh AK (2012) The Growing Role of Emergency Departments in Hospital Admissions. N Engl J Med 367: 391-393.

2. Scotti E, Pietrantonio F (2013) The hospital Internal Medicine specialist today: a literature review and strength, weaknesses, opportunity, threats (SWOT) analysis to develop a working proposal. It J Med 7: 278-286

3. Nardi R, Scanelli G, Borioni D, Grandi M, Sacchetti C, et al. (2007) The assessment of complexity in internal medicine patients. The FADOI Medicomplex Study. Eur J Int Med 18: 283-287. 
Citation: Pietrantonio F, Scotti E (2018) Who Makes the Diagnosis? A Retrospective Observational Study Comparing the Emergency Department Initial Diagnosis and the Internal Medicine Discharge Diagnosis. Int J Community Fam Med 3: 137. https://doi.org/10.15344/2456-3498/2018/137

Page 5 of 5

4. Pietrantonio F, Scotti E (2016) Internal medicine network: Is it a newsworthy idea?. Eur J Intern Med 35: e18-e19.

5. Chesi G, Nardi R (2013) Severity Stratification of patients hospitalized on the Internal Medicine ward: work in progress Ital J Med 7: 231-233.

6. Internal Medicine and hospital organization based on treatment intensity: the position of FADOI Emilia Romagna (2012) (Medicina Interna e organizzazione ospedaliera per intensità di cure: la posizione di FADOI Emilia Romagna) Ital J Med 6: 72-74.

7. Pietrantonio F, Piasini L, Spandonaro F (2016) Internal Medicine and emergency admissions: from a national Hospital Discharge Records (SDO) study to a regional analysis. Italian Journal of Medicine Italian Journal of Medicine 10: 157-167.

8. Peng A, Rohacek M, Ackermann S, Ilsemann-Karakoumis J, Ghanim L, et al. (2015) The proportion of correct diagnoses is low in emergency patients with nonspecific complaints presenting to the emergency department. Swiss Med Wkly 145: w14121

9. Wahner-Roedler DL, Chaliki SS, Bauer BA, Bundrick JB, Bergstrom LR, et al. (2007) Who makes the diagnosis? The role of clinical skills and diagnostic test results. Eval Clin Pract 13: 321-325

10. Schiff GD, Hasan O, Kim S, Abrams R, Cosby K, et al. (2009) Diagnostic Error in Medicine: Analysis of 583 Physician-Reported Errors. Arch Intern Med 169: 1881-1887.

11. Nemec M, Koller MT, Nickel CH, Maile S, Winterhalder C, et al. (2010) Patients presenting to the emergency department with non-specific complaints: the Basel Non-specific Complaints (BANC) study. Acad Emerg Med 17: 284-292.

12. Kulstad EB, Sikka R, Sweis RT, Kelley KM, Rzechula KH, et al. (2010) $\mathrm{ED}$ overcrowding is associated with an increased frequency of medication errors. Am J Emerg Med 28: 304-309.

13. Heuer JF, Gruschka D, Crozier TA, Bleckmann A, Plock E, et al. (2012) Accuracy of prehospital diagnoses by emergency physicians: comparison with discharge diagnosis. Eur J Emerg Med 19: 292-296.

14. Pietrantonio F, Orlandini F, Moriconi L, La Regina M (2015) Acute Complex Care Model: An organizational approach for the medical care of hospitalized acute complex patients. Eur J Intern Med 26: 759-765.

15. Tozzi VD, Longo F, Pacileo G, Salvatore D, Pinelli N, et al. (2014) PDTA standard per le patologie croniche. $11 \mathrm{ASL}$ a confronto per la gestione della rete dei servizi per BPCO, tumore polmonare, ictus, scompenso cardiaco e artrite reumatoide. Biblioteca dell'economia d'azienda. Cergas-Bocconi Fiaso. Milano: Edizioni Egea.

16. Pietrantonio F, Scotti E (2017) Internal medicine network: a new way of thinking hospital-territory integration and public-private partnership. It $J$ Med 11: 85-94

17. Sprivulis PC, Da Silva JA, Jacobs IG, Frazer AR, Jelinek GA, et al. (2006) The association between hospital overcrowding and mortality among patients admitted via Western Australian emergency departments. Med J Aust 184: 208-212. 